\title{
Muon Spin Relaxation in Ferromagnets: Theoretical Results for Paramagnetic EuO and EuS
}

S W Lovesey and E Engdahl

Novemebr 1994 
DRAL is part of the Engineering and Physical

\section{Sciences Research Council}

The Engineering and Physical Sciences Research Council does not accept any responsibility for loss or damage arising from the use of information contained in any of its reports or in any communication about its tests or investigations 


\title{
Muon Spin Relaxation in Ferromagnets: \\ Theoretical Results for Paramagnetic EuO and EuS
}

\author{
Stephen W. Lovesey and Erik Engdahl \\ DRAL Rutherford Appleton Laboratory, Oxon OX11 0QX, England, U.K.
}

\begin{abstract}
The relaxation rate for depolarization of a positive muon implanted in an isotropic magnetic salt with ferromagnetic exchange interactions is studied theoretically, on the basis of the coupled-mode theory of critical and paramagnetic spin fluctuations and a full numerical evaluation of the dipole field experienced by the muon. The main findings from studies of realistic models of EuO and EuS are (a) a significant dependence of the relaxation rate, $\lambda$, on the assumed position of the implanted muon and (b) a monotonic temperature dependence, with $\lambda \sim \xi^{3 / 2}$ in the approach to the critical temperature at which the correlation length, $\xi$, diverges. In contrast, previous results for a model of an isotropic magnet with an antiferromagnetic exchange, $\mathrm{RbMnF}_{3}$, show that $\lambda$ for this magnet is not a monotonic function of the temperature, and in the precursor region to $T_{\mathrm{c}} \lambda$ increases with decreasing temperature with a power law behaviour $\lambda \sim \xi^{1 / 2}$. The calculated values of $\lambda$ for EuO are consistent with data from preliminary experiments on the same salt.
\end{abstract}





\section{(1.) Introduction}

Several experimental studies have demonstrated that measurements of the depolarization of positive muons implanted in magnetic materials have the potential to provide useful information, at an atomic level of detail, on the fluctuations of the magnetic moments; see, for example, Cox (1987) and Dalmas de Réotier et al. (1994). In a previous paper we provided a comprehensive theoretical investigation of relaxation in the paramagnetic phase of an antiferromagnetically coupled material (Lovesey et al. 1994). The present paper reports findings from a similar, comprehensive investigation of muon relaxation in isotropic, ferromagnetically coupled systems. Results for two materials, EuO and EuS, are provided.

The overall plan of the work is the same as that used to study the antiferromagnetically coupled salt, $\mathrm{RbMnF}_{3}$, namely, a complete numerical evaluation of the spatial Fourier transform of the dipole field between the muon moment and the atomic moments, and a description of critical and paramagnetic fluctuations of the atomic moments from a full version of the coupled-mode theory. In view of the strong similarities in the work for the two types of magnetic salts the background to the methods given here is very brief; the reader interested in these matters is referred to Lovesey et al. (1994).

Turning to the results of our work based on realistic models of EuO and EuS - an isotropic Heisenberg magnet with exchange interactions out to the second shell of neighbouring spins - we find that the magnitude and temperature dependence of the relaxation rate, $\lambda$, depend on the position assumed for the implanted muon. A similar finding was obtained in the study of $\mathrm{RbMnF}_{3}$. For the latter material, $\lambda$ is not a monotonic function of the temperature. In contrast, the relaxation rates for $\mathrm{EuO}$ and $\mathrm{EuS}$ are found to be monotonic functions of the temperature, cf. Table (1). In the approach to the critical temperature $\lambda$ increases, and the temperature dependence, expressed in terms of the correlation 
length, $\xi$, is provided by $\xi^{z-1}$ where the dynamical critical exponent $z=5 / 2(3 / 2)$ for a ferromagnetically (antiferromagnetically) coupled material.

An expression for $\lambda$ given in $\S 2$ is based on the assumption that the contact interaction is negligible compared to the dipole interaction (a full theory that includes both the dipole and contact interactions is reviewed by Lovesey et al. 1994). The behaviour of $\lambda$ in the critical region is the subject of $\S 3$. Results for the paramagnetic region and provided in $\S 4$, together with results from an investigation of the dependence of $\lambda$ on the position assumed for the implanted positive muon. Our findings are discussed in $\$ 5$.

\section{(2.) Muon Relaxation Rate}

A derivation of a formula for the relaxation rate, $\lambda$, based on Fermi's Gold Rule for transition rates, is reviewed by Lovesey et al. (1994) Here, we record the formula for a multidomain sample, in which domains are randomly oriented, so it is appropriate to average $\lambda$ over orientations of the muon spin axes relative to the crystal axes. If the magnetic field acting on the muon is $\mathbf{B}$, and its thermal average $\langle\mathbf{B}\rangle=0\left(T>T_{\mathrm{c}}\right)$, then the zero-field relaxation rate is determined from,

$$
\lambda=\frac{1}{3}\left(g_{\mu} \mu_{\mathrm{N}}\right)^{2} \int_{-\infty}^{\infty} \mathrm{d} t\left\langle\mathbf{B}^{+}(0) \cdot \mathbf{B}(t)\right\rangle
$$

where $\mathbf{B}(t)$ is the standard Heisenberg representation of the operator $\mathbf{B}$.

In the present case, it is further assumed that the only contribution to $\mathbf{B}$ is provided by the dipole interaction between atomic moments, $-g \mu_{\mathrm{B}} \mathbf{S}$, on a lattice and the muon moment. The dynamical properties of the atomic moments are described by a Kubo spin relaxation function, $F(\mathbf{k}, \omega)$, where $\mathbf{k}$ and $\omega$ are wave vector and frequency variables. One then finds, 


$$
\lambda=\left(\frac{2 \pi}{3}\right)\left(g g_{\mu} \mu_{\mathrm{N}} \mu_{\mathrm{B}} / \nu_{\mathrm{o}}\right)^{2}\left(T / N^{2}\right) \sum_{\mathbf{k}} \chi(\mathbf{k}) F(\mathbf{k}, 0) \sum_{\alpha \beta}\left|\nu_{\mathrm{o}} D^{\alpha \beta}(\mathbf{k})\right|^{2} .
$$

In this expression, $\chi(\mathbf{k})$ is the isothermal susceptibility, and $D^{\alpha \beta}(\mathbf{k})$ is the spatial Fourier transform of the dipole field. The latter depends on the lattice structure for the atomic moments, and the position of the implanted muon. Various properties of $D^{\alpha \beta}(\mathbf{k})$ are summarized in an appendix.

In the following section (2.2) is evaluated for temperatures $T \rightarrow T_{\mathrm{c}}$. For higher temperatures $\left(T>1.30 T_{\mathrm{c}}\right.$ ) we have evaluated (2.2) numerically using values of $F(\mathbf{k}, 0)$ obtained from the coupled-mode theory of critical and paramagnetic spin fluctuations.

\section{(3.) Critical Values of $\lambda$}

In the limit $T \rightarrow T_{\mathrm{c}}$ the isothermal susceptibility, $\chi(\mathbf{k})$, has a large peak at $k=0$, where it is of order $\left(1 / \mathrm{K}^{2}\right)$ and $\mathrm{K}$ is the inverse correlation length ( $\kappa=0$ at $T_{\mathrm{c}}$ ). We have shown that the spherical average of the spatial Fourier transform of the dipole field is a slowly varying function of $k$ for $\mathbf{k}$ in the vicinity of the Brillouin zone centre. These observations lead us to the result, for $T \rightarrow T_{\mathrm{c}}$,

$$
\lambda=\frac{2 \pi}{3}\left(g g_{\mu} \mu_{\mathrm{N}} \mu_{\mathrm{B}} / \nu_{\mathrm{o}}\right)^{2} \sum_{\alpha \beta}\left\{\left|\nu_{\mathrm{o}} D^{\alpha \beta}(\mathbf{k})\right|^{2}\right\}_{\mathrm{av} .}\left(T_{c} / N^{2}\right) \sum_{\mathbf{k}} \chi(\mathbf{k}) F(\mathbf{k}, 0)
$$

Here, the curly brackets denote an average of the enclosed quantity over the directions of $\mathbf{k}$ followed by the limit $k \rightarrow 0$. Further progress is made by using the Ornstein-Zernike form for the susceptibility, and the Markovian approximation for the relaxation function.

Inserting in (3.1) the values, 


$$
\chi(k)=(N / 2 j)\left(\kappa^{2}+k^{2}\right)^{-1},
$$

and,

$$
F(k, 0)=\{\pi \Gamma(k)\}^{-1},
$$

where $\Gamma(k)$ is the decay rate estimated from coupled-mode theory, namely,

$$
\Gamma(k)=\left(T j v_{\mathrm{o}} / 24 \pi\right)^{1 / 2} k^{2}\left(k^{2}+\kappa^{2}\right) / \kappa^{3 / 2},
$$

one arrives at,

$$
\lambda=0.115\left(g g_{\mu} \mu_{\mathrm{N}} \mu_{\mathrm{B}} / \nu_{\mathrm{o}}\right)^{2} \sum_{\alpha \beta}\left|\nu_{\mathrm{o}} D^{\alpha \beta}(0)\right|^{2}\left(T_{c} \nu_{\mathrm{o}}\right)^{1 / 2} /(j \kappa)^{3 / 2} .
$$

In these expressions, $v_{0}$ is the volume of a unit cell, and $j=a^{2}\left(J_{1}+J_{2}\right)$ where $J_{1}$ and $J_{2}$ are the two significant exchange parameters between nearest and next nearest magnetic atoms. We conclude that, for an isotropic ferromagnet the muon relaxation rate increases on approaching the critical temperature from above, and it grows with decreasing temperature as $(1 / \kappa)^{z-1}$ where $z$ is the dynamical critical exponent (Lovesey et al. 1992, Lovesey et al. 1994).

Various properties of the f.c.c. magnetic salts EuO and EuS are summarized by Lovesey and Trohidou (1991). Employing the appropriate values in (3.5) we find, $T \rightarrow T_{\mathrm{c}}$,

$$
\lambda(\mathrm{EuO})=\left\{1.34 /(a \kappa)^{3 / 2}\right\} \mu \mathrm{s}^{-1},
$$


where $a$ is the unit cell side. A similar expression is obtained for EuS apart from the numerical constant which has the value 4.06. In arriving at the result (3.6) we have exploited the result,

$$
\sum_{\alpha \beta}\left|v_{\mathrm{o}} D^{\alpha \beta}(0)\right|^{2}=6(4 \pi / 3)^{2}
$$

which is valid if the muon occupies a site of high symmetry; see appendix A. Experimental data for $\kappa$ reviewed by Als-Neilsen (1974) are well represented by,

$$
a \kappa=3.11\left(\frac{T}{T_{\mathrm{c}}}-1\right)^{0.70}
$$

This representation is good for both EuO and EuS for $T<1.3 T_{\mathrm{c}}$. Values of $\lambda$ derived from (3.6) and (3.8) are consistent with previous estimates (Lovesey et al. 1992).

For higher temperatures, the arguments used in the foregoing paragraphs are no longer sound, and full numerical evaluations of the dipole field and the spin relaxation function are called for. Results from such calculations are summarized in the following section.

\section{(4.) Paramagnetic Fluctuations}

In $\S 3$ we exploited the simplification in the evaluation of (2.2) that arises in the critical region from the concentration of weight in the kernel at the centre of the Brillouin zone. Outside the narrow band of temperatures in which the corresponding result for $\lambda$, equation (3.5), can be used with confidence it is necessary to (a) calculate the spin response function throughout the Brillouin zone and (b) calculate the spatial Fourier transform of the dipole field at the site of the implanted muon. Armed with this data, at a sufficient number of points in the zone, the integration over the Brillouin zone in (2.2) is readily accomplished. 
Item (b) has been discussed by Lovesey et al. (1994). Additional information on the dipole fields in general and specifically for EuO and EuS is gathered in an appendix to this paper. Regarding item (a), the spin response function is derived from a full version of coupled-mode theory. Details of the theory and its application to EuO and EuS are covered by Cuccoli et al. (1989).

In view of the extensive background to the two items required to obtain reliable values for $\lambda$ from (2.2) we will here move immediately to the findings of our numerical studies. Results for $\lambda$, in units of $\mu \mathrm{s}^{-1}$, are listed in Table (1) for $\left(T / T_{\mathfrak{c}}\right) \geq 1.3$. Data is provided for two muon sites. One is a reasonable first guess, namely, $\delta_{1}=a 0.25(1,1,1)$. The second site is chosen to illustrate the variation in magnitude and the temperature dependence with changes in $\delta$. Before moving to a fuller account of this dependence, we note that $\lambda$ is a monotonic function of the temperature in contrast to our previous findings for an antiferromagnetically coupled salt.

Fig. 1 displays $\lambda$ when the muon site is varied along the main diagonal of the chemical unit cell of EuO. The parameter $x=0.25$ corresponds to our first guess of the muon location, whereas $x=0.50$ would mean that muon site coincides with on the oxygen ions. Near $x=0.25, \lambda$ shows a minimum irrespective of temperature. The curves in Fig. 1 for the two temperature $\left(T / T_{\mathfrak{c}}\right)=1.3$ and 2.0 are not parallel. This is due to different width of the $\mathbf{k}=0$ peak of the van Hove response function,

$S(\mathbf{k}, \omega=0)=(T / N) \chi(\mathbf{k}) F(\mathbf{k}, 0)$.

If one would have done the continuum limit approximation of the dipolar structure factor, as in the previous section, the curves would become parallel 


\section{(5.) Discussion}

The theoretical findings reported here for muon spin relaxation rates in ferromagnetically (F) coupled paramagnets complement previous work on antiferromagnetically (AF) coupled magnets (Lovesey et al. 1994). In the process, we have also added to our knowledge about the behaviour of the dipole field between the implanted muon and atomic spins, and investigated another method for the numerical computation of the field.

Our analytical and numerical work shows that for $(F)$ the relaxation rate, $\lambda$, decreases monotonically with temperature as the temperature is increased from the critical region, where $\lambda$ is proportional to $\xi^{3 / 2}$ and $\xi$ is the correlation length. At very high temperatures the relaxation rates for our two examples, which are models of EuO and EuS, are almost the same size. On the other hand, in the approach to the critical region the relaxation rates for EuS exceed those for EuO by a factor that is of the order of $2-3$ (for the same values of $T / T_{\mathrm{c}}$ ). The values of $\lambda$ given in Table (1) for EuO are consistent with results from preliminary experiments (Hartmann, 1994).

Looking at our results for (F) and (AF) magnets two findings merit some comment. First, for both magnets, $\lambda$ depends to a significant degree on the location in the chemical unit cell of the implanted muon. So, a quantitative analysis of $\mu$ SR signals demands accurate information on the positions of the implanted muon. Secondly, the temperature dependence of $\lambda$, as a function of the reduced variables $\left(T / T_{\mathrm{c}}\right)$, is different for $(\mathrm{F})$ and $(\mathrm{AF})$ systems. In the critical region $\lambda \propto \xi^{z-1}$ with $z=5 / 2(\mathrm{~F})$ and $z=3 / 2(\mathrm{AF})$. At higher temperatures, $\lambda$ decreases monotonically for the (F) system while for (AF) it reaches a minimum before recovering to a value at infinite temperature that is comparable in size to values predicted in the precursor to the critical region, say $\left(T / T_{\mathrm{c}}\right) \sim 1.10$. We have verified that differences in $\lambda\left(T / T_{\mathrm{c}}\right)$ for the two systems arises from differences in, 


$$
\sum_{\mathbf{k}} \chi(\mathbf{k}) F(\mathbf{k}, \omega=0),
$$

as a function of $\left(T / T_{\mathrm{c}}\right)$ for $(\mathrm{F})$ and $(\mathrm{AF})$ systems. A possibly interesting form of plausibility argument is obtained by use of a gaussian approximation for $F(\mathbf{k}, \omega)$, in which case the foregoing function is proportional to,

$$
\sum_{\mathbf{k}} \chi^{3 / 2}(\mathbf{k}) /\left(1-\gamma_{\mathbf{k}}\right)^{1 / 2}
$$

Evaluation of this quantity, using the appropriate forms of $\chi(\mathbf{k})$, as a function of $\left(T / T_{\mathrm{c}}\right)$ reveals features similar to the ones obtained in our comprehensive calculation based on coupled-mode theory.

Finally, it remains to say that, in the present study we have undertaken the same tests of our numerical work as we did in the previous study of a model of $\mathrm{RbMnF}_{3}$ (Lovesey et al. 1994).

\section{Acknowledgements}

We are grateful to Dr. S. F. J. Cox for his interest in our work on the theory of $\mu \mathrm{SR}$, and Dr. O. Hartmann for making available experimental results for EuO. 


\section{References}

Als-Nielsen, J., Phase Transitions and Critical Penomena, Vol. 5a (Academic Press, London 1974).

Cox, S.F.J., J. Phys.:Condens. Matter C20 (1987) 3187.

Cuccoli, A., Tognetti, V., and Lovesey, S.W., Phys. Rev. B39 (1989) 2619.

Dalmas de Réotier, P., Yaouanc, A., and Frey, E., Phys. Rev. B50 (1994) 3033.

Hartmann, O. (1994) private communication.

Kittel, C., Introduction to Solid State Physics, Appendix B (John Wiley, New York, 1986).

Lovesey, S.W., and Trohidou, K.N., J. Phys.: Condens. Matter 3 (1991) 1827.

Lovesey, S.W., Trohidou, K.N., and Karlsson, E.B., J. Phys.: Condens. Matter 4 (1992) 2061 : ibid 4 (1992) 9489.

Lovesey, S.W., Balcar, E., and Cuccoli, A., submitted to J. Phys.: Condens. Matter (1994)

\section{Figure Caption}

Values of the muon relaxation rate for two values of the reduced temperature, $\left(T / T_{\mathrm{c}}\right)=1.3$ and 2.0, are shown as a function of position of the muon $\delta=\operatorname{ax}(1,1,1)$ where the parameter $x$ extends up to 0.4. Data for $\left(T / T_{\mathrm{c}}\right)=1.3(2.0)$ are denoted by x (o). Results are for our model of EuO. 


\section{Table 1}

\begin{tabular}{lll|ll}
$T / T_{\mathrm{c}}$ & \multicolumn{2}{c}{ EuO } & \multicolumn{2}{c}{ EuS } \\
& $\lambda(1) \mu \mathrm{s}^{-1}$ & $\lambda(2) / \lambda(1)$ & $\lambda(1) \mu \mathrm{s}^{-1}$ & $\lambda(2) / \lambda(1)$ \\
1.3 & 1.51 & 1.42 & 3.14 & 1.53 \\
1.5 & 1.35 & 1.35 & 2.44 & 1.43 \\
2.0 & 1.24 & 1.27 & 1.86 & 1.32 \\
4.0 & 1.21 & 1.20 & 1.47 & 1.22 \\
$\infty$ & 1.22 & 1.16 & 1.27 & 1.16
\end{tabular}

Representative values of muon relaxation rates in isotropic ferromagnets. $\lambda(j)$ corresponds to a muon site $\delta_{j}$, and $\delta_{1}=a 0.25(1,1,1)$ and $\delta_{2}=a 0.35(1,1,1)$. 


\section{Appendix}

\section{(A.1) Fourier transform of dipolar field}

The spatial transform of the dipolar field is given by,

$$
D^{\alpha \beta}(\mathbf{k})=\sum_{a}\left(1 / R_{a}^{3}\right)\left(3 \hat{R}_{a}^{\alpha} \hat{R}_{a}^{\beta}-\delta_{\alpha \beta}\right) \exp \left(\mathrm{i} \mathbf{k} \cdot \mathbf{R}_{a}\right)
$$

where $\mathbf{R}_{a}=\mathbf{R}_{\mathrm{o}}+\mathbf{a}=-\delta+\mathbf{a}$ is the vector from the $\mu^{+}$to the a'th magnetic ion, $\hat{\mathbf{R}}_{a}=\mathbf{R}_{a} / R_{a}$ and $R_{a}=\left|\mathbf{R}_{a}\right|$. (NB, the definition in (A.1) differs from that used by Lovesey et al. 1994 by the presence of an extra trivial phase factor $\exp (-\mathbf{i k} \cdot \boldsymbol{\delta})$.) The vectors $\{\mathbf{a}\}$ exhaust the direct lattice with respect to a chosen lattice origin $\mathbf{a}=0$, and $\delta$ gives the location of the muon with respect to the same lattice origin. $\alpha$ and $\beta$ label Cartesian components. Although the sum in (A.1) is unconditionally convergent, it is rather slowly convergent. For numerical computation it is suitable to improve the convergence by employing the Ewald summation method; see, for example, Kittel (1986). The dipolar sum then becomes,

$$
\begin{aligned}
D^{\alpha \beta}(\mathbf{k})= & \rho^{3} \sum_{a} H_{\alpha \beta}\left(\rho \mathbf{R}_{a}\right) \exp \left(\mathrm{ik} \cdot \mathbf{R}_{a}\right)-\frac{4 \pi}{v_{\mathrm{o}}} \hat{k}^{\alpha} \hat{k}^{\beta} \exp \left(-k^{2} / 4 \rho^{2}\right) \\
& -\frac{\pi}{\rho^{2} v_{\mathrm{o}}} \sum_{\mathbf{K}}\left(K^{\alpha}+k^{\alpha}\right)\left(K^{\beta}+k^{\beta}\right) Q\left[\frac{(\mathbf{K}+\mathbf{k})^{2}}{4 \rho^{2}}\right] \exp \left(-\mathrm{i} \mathbf{K} \cdot \mathbf{R}_{\mathrm{o}}\right),
\end{aligned}
$$

where,

$$
H_{\alpha \beta}(\mathbf{r})=\frac{\partial}{\partial r^{\alpha} \partial r^{\beta}} H(r), \text { and } H(r)=\frac{2}{\sqrt{\pi}} \frac{1}{r} \int_{r}^{\infty} \exp \left(-r^{2}\right) \mathrm{d} r=\frac{1}{r} \operatorname{erfc}(r)
$$

$Q(k)=\frac{1}{k} \exp (-k)$, and $\hat{\mathbf{k}}=\mathbf{k} /|\mathbf{k}|$. 
The notation $\sum_{\mathbf{K}}^{\prime}$ indicates that the term $\mathbf{K}=\mathbf{0}$ is excluded in the reciprocal lattice summation. $\rho$ is a free parameter that is discussed below.

An interpretation of the Ewald summation is that part of the summation is done in the direct lattice and part in the reciprocal lattice. The free parameter $\rho$ determines the relative weight of these two summations. The limit $\rho \rightarrow 0$ corresponds to the original summation in (A.1), whereas $\rho \rightarrow \infty$ corresponds to a summation entirely in the reciprocal lattice.

The formula (A.2) has been implemented by a computer program for several lattice structures. In the program, the summations are grouped into cubical shells centred at $\mathbf{a}=\mathbf{0}$ and $\mathbf{K}=\mathbf{0}$ respectively. Cubic shells are added until the contribution from the latest shell is found to be smaller than a chosen small value. This value should be small enough to avoid spurious convergence since sometimes a shell-grouped series is monotonically convergent rather than alternating.

In most cases, the free parameter was chosen $\rho=0.5$ which means roughly an equal number of cubic shells in the summation in the direct and reciprocal lattices.

\section{(A.2) Dipolar structure factor}

The dipolar structure factor occurring in (2.2) is,

$$
G(\mathbf{k})=\sum_{\alpha \beta}\left|D^{\alpha \beta}(\mathbf{k})\right|^{2}
$$

For a general $\mu^{+}$location, $\delta$, of no particular symmetry, the function $G(\mathbf{k})$ shows no symmetry with respect to $\mathbf{k}$ except for the trivial $G(\mathbf{k})=G(-\mathbf{k})$. Therefore, 
one generally has to compute $G(\mathbf{k})$ for a sufficiently fine mesh in the entire first Brillouin zone (BZ). It is not necessary that this mesh follows the structure of the reciprocal lattice. We have chosen a mesh consisting of $N$ points in a s.c. type mesh in the first BZ.

Sometimes, the concomitant factor $\chi(\mathbf{k}) F(\mathbf{k}, 0)$ in (2.2) displays a symmetry such that it can be reduced to an irreducible polyhedra in the first BZ. In particular, this happens in the absence of anisotropic interactions in the Hamiltonian and use of spherical model susceptibility. Then the summation can be grouped according to,

$$
\frac{1}{N^{2}} \sum_{\mathbf{k}}^{\mathrm{BZ}} \chi(\mathbf{k}) F(\mathbf{k}, 0) G(\mathbf{k})=\frac{1}{N^{2}} \sum_{\mathbf{k}}^{\mathrm{irr}} \chi(\mathbf{k}) F(\mathbf{k}, 0) G^{\prime}(\mathbf{k})
$$

where $G^{\prime}(\mathbf{k})=\sum_{\mathbf{k} \in J(\mathbf{k})} G(\mathbf{k})$ and $I(\mathbf{k})$ is the full set of wave vectors that reduces to the particular wave vector $\mathbf{k}$ in the irreducible polyhedra of $\chi(\mathbf{k}) F(\mathbf{k}, 0)$.

It has been verified that the results of the present program for the dipolar structure factor agree with those obtained previously by Lovesey et al. 1994 .

\section{(A.3) Continuum limit and spherical average of dipolar structure factor}

For $T$ in the precursor region $T \rightarrow T_{\mathrm{c}}^{+}$, the van Hove response function $S(\mathbf{k}, \omega=0)=(T / N) \chi(\mathbf{k}) F(\mathbf{k}, 0)$ becomes strongly peaked about $\mathbf{k}=0$. It is therefore interesting to study $D^{\alpha \beta}(\mathbf{k})$ around $\mathbf{k}=0$. This is most conveniently done by (A.2) in the $\rho \rightarrow \infty$ limit. We find,

$$
D^{\alpha \beta}(\mathbf{k})=-\frac{4 \pi}{v_{\mathrm{o}}}\left(\hat{k}^{\alpha} \hat{k}^{\beta}-C^{\alpha \beta}(\mathbf{k})\right)
$$


where,

$$
C^{\alpha \beta}(\mathbf{k})=-\sum_{\mathbf{K}}{ }^{\prime} \frac{\left(K^{\alpha}+k^{\alpha}\right)\left(K^{\beta}+k^{\beta}\right)}{(\mathbf{K}+\mathbf{k})^{2}} \exp \left(-\mathrm{i} \mathbf{K} \cdot \mathbf{R}_{\mathrm{o}}\right) .
$$

Since the $\mathbf{K}=0$ term is extracted from the left hand side, $C^{\alpha \beta}(\mathbf{k})$ is analytic at $\mathbf{k}=0$. One finds the symmetry rules $D^{\alpha \beta}(\mathbf{k})=D^{\beta \alpha}(\mathbf{k})$ and $D^{\alpha \beta}(-\mathbf{k})=\left(D^{\alpha \beta}(\mathbf{k})\right)^{*}$. This corresponds to $C^{\alpha \beta}(\mathbf{k})=C^{\beta \alpha}(\mathbf{k})$ and $C^{\alpha \beta}(-\mathbf{k})=\left(C^{\alpha \beta}(\mathbf{k})\right)^{*}$. There is also the summation rule $\sum_{\alpha} D^{\alpha \alpha}(\mathbf{k})=0$, corresponding to $\sum_{\alpha} C^{\alpha \alpha}(\mathbf{k})=1$. Since, Re. $C^{\alpha \beta}(\mathbf{k})$ is even in $\mathbf{k}$ and $\operatorname{Im} . C^{\alpha \beta}(\mathbf{k})$ is odd, $C^{\alpha \beta}(\mathbf{k})$ expanded up to second order in $\mathbf{k}$ can be written,

$$
C^{\alpha \beta}(\mathbf{k})=c^{\alpha \beta}+\sum_{\gamma} \mathrm{i} d_{\gamma}^{\alpha \beta} k^{\gamma}+\frac{1}{2} \sum_{\gamma \delta} e_{\gamma \delta}^{\alpha \beta} k^{\gamma} k^{\delta}
$$

where $c^{\alpha \beta}, d_{\gamma}^{\alpha \beta}$ and $e_{\gamma \delta}^{\alpha \beta}$ are real. The sum rule becomes $\sum_{\alpha} c^{\alpha \alpha}=1, \sum_{\alpha} d_{\gamma}^{\alpha \alpha}=0$ and $\sum_{\alpha} e_{\gamma \delta}^{\alpha \alpha}=0$

The continuum limit $C^{\alpha \beta}(\mathbf{k} \rightarrow 0)$ was computed by first using (A.2) with $\mathbf{k}_{1}=(2 \pi / a)(0,0,1) 10^{-3}$ and $\mathbf{k}_{2}=(2 \pi / a)(0,0,2) 10^{-3}$. Use of (A.7) then gives the estimate,

$$
c^{\alpha \beta}=\operatorname{Re} . C^{\alpha \beta}\left(\mathbf{k}_{1}\right)-\frac{\operatorname{Re} \cdot C^{\alpha \beta}\left(\mathbf{k}_{2}\right)-\operatorname{Re} \cdot C^{\alpha \beta}\left(\mathbf{k}_{1}\right)}{k_{2}^{2}-k_{1}^{2}} k_{1}^{2} .
$$

In the continuum limit we find,

$$
D^{\alpha \beta}(\mathbf{k} \rightarrow 0)=-\frac{4 \pi}{v_{\mathrm{o}}}\left(\hat{k}^{\alpha} \hat{k}^{\beta}-c^{\alpha \beta}\right),
$$


which is non-analytic at $\mathbf{k}=0$ having constant values along any straight ray towards $\mathbf{k}=0$. The corresponding structure factor is,

$$
G(\mathbf{k} \rightarrow 0)=\left(\frac{4 \pi}{v_{\mathrm{o}}}\right)^{2}\left\{1-2 \sum_{\alpha \beta} \hat{k}^{\alpha} \hat{k}^{\beta} c^{\alpha \beta}+\sum_{\alpha \beta}\left(c^{\alpha \beta}\right)^{2}\right\} .
$$

Since the response function is isotropically peaked at $\mathbf{k}=0$, this structure factor could be substituted for its isotropic spherical average.

Using,

$$
\frac{1}{4 \pi} \int \mathrm{d} \hat{\mathbf{k}} \hat{k}^{\alpha} \hat{k}^{\beta}=\frac{1}{3} \delta_{\alpha \beta},
$$

and $\sum_{\alpha} c^{\alpha \alpha}=1$ gives,

$$
\{G(\mathbf{k})\}_{\mathrm{av} .}=\left(\frac{4 \pi}{v_{\mathrm{o}}}\right)^{2}\left[\frac{1}{3}+\sum_{\alpha \beta}\left(c^{\alpha \beta}\right)^{2}\right] .
$$

The curly brackets denote a spherical average over the directions of $\mathbf{k}$ followed by the limit $k \rightarrow 0$.

In particular, for a symmetric muon position, such as $\delta=\frac{1}{4} a(1,1,1)$, we recover the Holstein-Primakoff result $c^{\alpha \beta}=\frac{1}{3} \delta_{\alpha \beta}$. This gives for the structure factor the value $\left(\frac{4 \pi}{\nu_{\mathrm{o}}}\right)^{2} \frac{2}{3}$. 


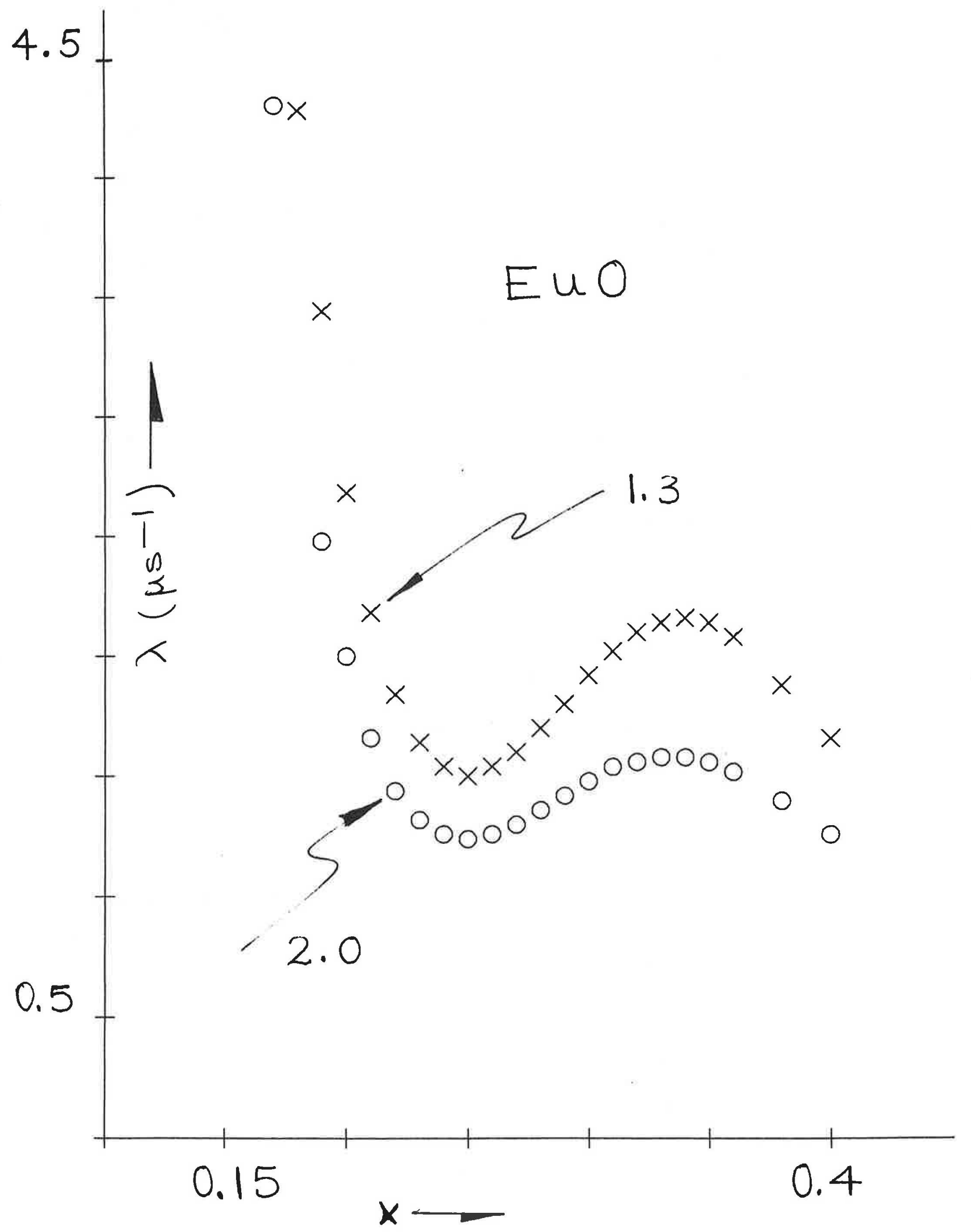

Fig. 1. 

